Hautarzt 2009 -60:857-858

DOI 10.1007/s00105-009-1863-5

Online publiziert: 25 . Oktober 2009

(c) Springer Medizin Verlag 2009

\author{
H. Hintner \\ Univ.-Klinik für Dermatologie der Paracelsus Medizinische Privatuniversität \\ (PMU) Salzburg/Salzburger Landeskliniken (SALK), Salzburg
}

\title{
Erkrankungen der einsehbaren Schleimhäute
}

\section{Liebe Leserinnen und Leser!}

Das Leitthemenheft „Erkrankungen der einsehbaren Schleimhäute" befasst sich schwerpunktmäßig mit Veränderungen an Mund- bzw. Genitalschleimhaut sowie zum Teil an den Konjunktiven. Das weite Spektrum machte es notwendig, beispielhaft einige Erkrankungsgruppen herauszugreifen.

Nicht selten hört man von Patientinnen und Patienten den Kommentar „Ich habe nicht gewusst, dass Veränderungen im Mund bzw. im Genitalbereich zum Hautfach gehören", und es ist leider noch keine Selbstverständlichkeit, dass Kolleginnen und Kollegen in der Spezialität Dermatovenerologie bei einer Gesamtuntersuchung jedes Mal auch die einsehbaren Schleimhäute mit beurteilen. Dabei fällt natürlich ins Kalkül, dass bei Erkrankungen der genannten Schleimhautregionen tatsächlich Kolleginnen und Kollegen in den Schwesterdisziplinen wie der Hals-, Nasen- und Ohrenheilkunde, der Zahnheilkunde bzw. Gynäkologie und Urologie konsultiert werden. Dies fordert zu interdisziplinärer Zusammenarbeit auf, die meist auch sehr gut funktioniert.

Eine eiserne Regel gilt bei Krankheitserscheinungen an den einsehbaren Schleimhäuten in derselben Weise wie für das gesamte Integument: Ist die Erkrankung klinisch nicht eindeutig zuzuordnen bzw. von vornherein bereits klar zu klassifizieren oder entspricht der Behandlungserfolg (bei entsprechender Compliance der Patientinnen und Patienten) nicht Ihren Erwartungen als behandelnde Ärztin oder behandelnder Arzt, dann ist eine Probeexzision (oft mit Immunfluoreszenz
- optischer Untersuchung bzw. sequenziell) eine Conditio sine qua non.

Der erosive Lichen ruber der Mundschleimhaut, der von Michael Sticherling in seinem Beitrag über die Schleimhautbeteiligung bei papulosquamösen Erkrankungen in bewährter Weise dargestellt ist, beschreibt in sich beispielhaft 2 besondere Eckpunkte der Schleimhauterkrankungen: Einerseits sieht man bei den betroffenen Patientinnen und Patienten die Veränderungen an der Mundschleimhaut (oder Genitalschleimhaut) beim alltäglichen Kontakt nicht (also eine kosmetisch nicht beeinträchtigende Erkrankung!), andererseits können die Erosionen sprechen, essen und trinken in einer Weise beeinträchtigen, dass die Kommunikation behindert und ein Gewichtsverlust die Folge ist.

Im Beitrag von Johann Beck-Mannagetta ist der wichtigste Punkt die Früherkennung von Vorstufen (Erythroleukoplakie) des häufigsten malignen Tumors der Mundschleimhaut, nämlich des Plattenepithelkarzinoms. Diagnostisch bedeutet immer noch die repräsentative Probeexzision das richtige Vorgehen, und auch therapeutisch ist die Operation mit möglichst vollständiger Entfernung von Tumorzellen die Therapie der Wahl. Zielführend wäre natürlich eine Aufklärungskampagne, dass Tabak und Alkohol ursächlich mit der Entstehung des Mundhöhlenkarzinoms in Verbindung stehen!

Die Arbeitsgruppe von Reinhard Kirnbauer behandelt in ihrem Beitrag über HPV-Infektionen der Mund- und Genitalschleimhaut die Möglichkeiten der primären Prävention durch HPV-Vakzine $\left(\right.$ Cervarix $^{\mathrm{TM}}$, Gardasil $\left.{ }^{\mathrm{TM}}\right)$. Dies ist ein 
heftig diskutiertes Thema und daher von großem Interesse. Auch die Entwicklung einer potenziellen Breitspektrumvakzine gegen mukosale Hoch- und Niedrig-Risiko-HPV-Typen wird angesprochen.

Susanna Fistarol und Peter Itin zeigen in einer beeindruckenden Übersicht die Bedeutung von Mundschleimhautveränderungen in der Diagnose des enormen Spektrums von Systemerkrankungen. Herausgreifen möchte ich z. B. das primäre Sjögren-Syndrom, das 3-4\% der über 50-jährigen Frauen trifft, oft sehr spät diagnostiziert wird und bei dem das Risiko für die Entwicklung eines lymphoretikulären Malignoms um das 44-Fache erhöht ist.

Martin Laimer bespricht die Mundund Genitalschleimhautbeteiligung sowie Bindehauterkrankungen bei blasenbildenden Dermatosen und stellt den teils fatalen Krankheitswert mit eindrucksvollem Bildmaterial dar. Bei der Therapie fasst er einen weiten Bogen von molekularbiologischen Ansätzen (z. B. Ex-vivo-Gentherapie) bei Genodermatosen bis hin zur äußerst teuren, aber sehr effektiven Kombinationstherapie von i.v. verabreichten Immunglobulinen sowie Rituximab.

Wussten Sie übrigens, was eine „strawberry gingivitis" ist?

Wenn nicht, lesen Sie die Beiträge in diesem Themenheft!
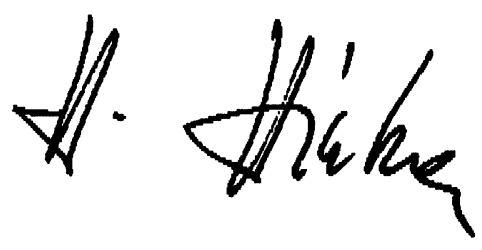

Ihr H. Hintner

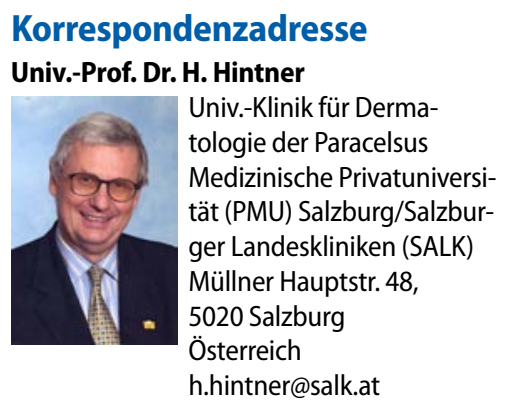

Interessenkonflikt. Der korrespondierende Autor gibt an, dass kein Interessenkonflikt besteht.

\section{Möchten Sie einen Beitrag für „Der Hautarzt" einreichen?}

Wir freuen uns, dass Sie unsere Zeitschrift "Der Hautarzt" mitgestalten möchten. Um Ihnen bei der Manuskripterstellung behilflich zu sein, haben wir für unsere Autoren Hinweise zusammengestellt, die Sie im Internet finden unter www.DerHautarzt.de (Für Autoren). Bitte senden Sie Ihren fertigen Beitrag an:

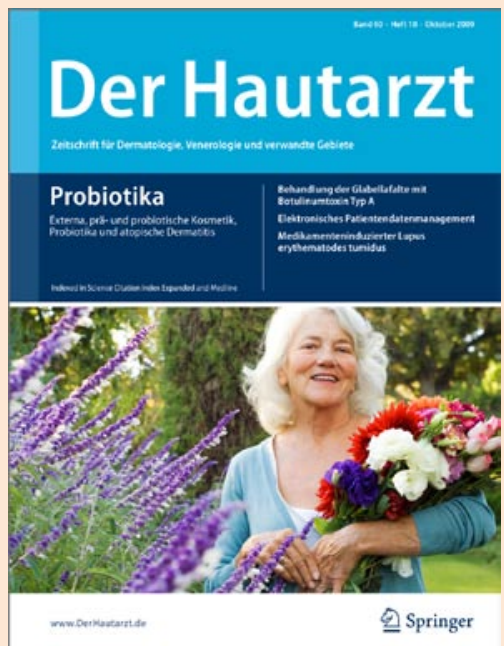

\section{Übersichten:}

Prof. Dr. Alexander Kapp

Klinik und Poliklinik für Dermatologie und Venerologie, Medizinische Hochschule Hannover, Ricklinger Straße 5, 30449 Hannover

Tel: +49-511-9246-232

Fax: +49-511-9246-234

freimooser.martina@mh-hannover.de

Originalien, Kasuistiken:

Prof. Dr. Thomas Ruzicka

Klinik und Poliklinik für Dermatologie, LMU München

\section{Anfragen an:}

Prof. Dr. Daniela Bruch-Gerharz

Hautklinik des Universitätsklinikums

Düsseldorf, Moorenstr. 5,

40225 Düsseldorf

Tel: +49-211-81-18328

Fax:+49-211-81-04905

s.gehrke@med.uni-duesseldorf.de

\author{
Weiterbildung • \\ Zertifizierte Fortbildung: \\ Anfragen an:
}

Prof. Dr. Michael Meurer Klinik und Poliklinik für Dermatologie an der Universitätsklinik Carl Gustav Carus, TU Dresden, Fetscherstr. 74, 01307 Dresden meurer@rcs.urz.tu-dresden.de

Prof. Dr. Rolf-Markus Szeimies Klinik für Haut-, Allergie-, Venen- und Umwelterkrankungen,

Knappschaftskrankenhaus

Recklinghausen, Klinikum Vest $\mathrm{GmbH}$, Dorstener Str. 151, 45657 Recklinghausen dermatologie@kk-recklinghausen.de

Wie lautet Ihre Diagnose?: Prof. Dr. Karin Scharffetter-Kochanek Universitätsklinik und Poliklinik für Dermatologie, Abteilung für Dermatologie und Allergologie, Maienweg 12, $89081 \mathrm{UIm}$ Tel: +49-731-500-21801 Fax: +49-731-500-21870

\section{In der Diskussion:}

Prof. Dr. Alexander Kapp/

Prof. Dr. Thomas Werfel

Klinik und Poliklinik für

Dermatologie und Venerologie, Medizinische Hochschule Hannover, Ricklinger Straße 5, 30449 Hannover freimooser.martina@mh-hannover.de

Leserforum:

Prof. Dr. Hans F. Merk

Klinik für Dermatologie und Allergologie, Universitätsklinikum der RWTH, Pauwelsstraße 30, 52057 Aachen Hans.Merk@post.rwth-aachen.de

Gesundheitsökonomie:

Prof. Dr. Michael Jünger

Klinik und Poliklinik für Hautkrankheiten, Klinikum der Ernst-MoritzArndt-Universität Greifswald, Fleischmannstraße 42-44, 17487 Greifswald juenger@uni-greifswald.de 